INPLASY

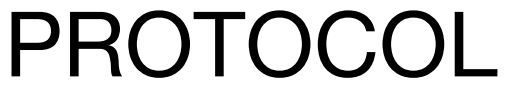

To cite: Li et al. Tripterygium wilfordii Hook.f. preparations (TWPs) for Rheumatoid

Arthritis (RA): An Overview of

Systematic Reviews. Inplasy

protocol 202180081. doi:

10.37766/inplasy2021.8.0081

Received: 21 August 2021

Published: 21 August 2021

Corresponding author:

Xing Liao

okfrom2008@hotmail.com

Author Affiliation:

Center for Evidence-based

Chinese Medicine, Institute of Basic Research in Clinical Medicine, China Academy of Chinese Medical Sciences, Beijing, China.

Support: National Natural Science Found.

Review Stage at time of this submission: Preliminary searches.

Conflicts of interest:

None declared.

\section{Tripterygium wilfordii Hook.f. preparations (TWPs) for Rheumatoid Arthritis (RA): An Overview of Systematic Reviews}

Li, H4; Hu, R²; Dai, Z3; Xu, S4; Liao, X5.

Review question / Objective: Since current systematic reviews (SRs) show that results of effectiveness on Tripterygium wilfordii Hook. F preparations for Rheumatoid Arthritis are inconsistent, the purpose of this study is to find the reasons of the disparity by comprehensively appraising the related SRs.

Condition being studied: Rheumatoid Arthritis (RA).

Information sources: Electronic bibliographic databases: PubMed, Embase, The Cochrane Library, China National Knowledge Infrastructure (CNKI), Wan Fang database CBM database and VIP database. Studies published in English and Chinese were included. In addition, we manually searched the references and relevant domestic journals, including the Rheumatism and Arthritis, Chinese Journal of Rheumatology, and the World Journal of Rheumatology.

INPLASY registration number: This protocol was registered with the International Platform of Registered Systematic Review and Meta-Analysis Protocols (INPLASY) on 21 August 2021 and was last updated on 21 August 2021 (registration number INPLASY202180081).

\section{INTRODUCTION}

Review question / Objective: Since current systematic reviews (SRs) show that results of effectiveness on Tripterygium wilfordii Hook. F preparations for Rheumatoid
Arthritis are inconsistent, the purpose of this study is to find the reasons of the disparity by comprehensively appraising the related SRs. 
Rationale: Rheumatoid arthritis (RA) is a deformity and dysfunction caused by synovitis that leads to destruction of the joint capsule and articular cartilage. Owing to the limitations of conventional treatments, patients with RA are often dissatisfied with the effect of treatment and have a poor quality of life. Tripterygium wilfordii Hook. F preparations(TWPs) are frequently used for the treatment of RA. This article was designed through an overview of systematic reviews (SRs), to investigate the safety and efficacy of TWHF for treating RA.

Condition being studied: Rheumatoid Arthritis (RA).

\section{METHODS}

Search strategy: We searched the following electronic bibliographic databases: PubMed, Embase, The Cochrane Library, China National Knowledge Infrastructure (CNKI), Wan Fang database CBM database and VIP database, using the keywords of TWPs, RA, and systematic review . Studies published in English and Chinese were included. In addition, we manually searched the references and relevant domestic journals, including the Rheumatism and Arthritis, Chinese Journal of Rheumatology, and the World Journal of Rheumatology.

Participant or population: Participants: subjects diagnosed with RA.

Intervention: Interventions: Any type of TWHs with or without routine treatments.

Comparator: Comparison intervention: Routine treatments, such as drug therapy, routine activities, and other treatment, no therapy, placebo.

Study designs to be included: SRs containing more than one randomized controlled trial (RCT)will be included.

Eligibility criteria: Inclusion criteria were established as follows: i) types of study: SRs containing more than one randomized controlled trial (RCT); ii) participants: subjects diagnosed with RA; iii) interventions: Any type of TWHs with or without routine treatments; iv) comparison intervention: Routine treatments, such as drug therapy, routine activities, and other treatment, no therapy, placebo.

Information sources: Electronic bibliographic databases: PubMed, Embase, The Cochrane Library, China National Knowledge Infrastructure (CNKI), Wan Fang database CBM database and VIP database. Studies published in English and Chinese were included. In addition, we manually searched the references and relevant domestic journals, including the Rheumatism and Arthritis, Chinese Journal of Rheumatology, and the World Journal of Rheumatology.

Main outcome(s): SJC(swollen joint count),MS(morning stiffness),GS(grip strength),RF(Rheumatoid Factor), ESR (Erythrocyte Sedimentation Rate), CRP (Creactive protein) ,TJC (tender joint count), TNF-a (Tumor Necrosis Factor-Alpha), CTE (Clinical Treatment Efficacy), ACR20/50/70, AEs(Adverse Events).

Additional outcome(s): Interleukin 1=IL-1.;

Interleukin $6=$ IL 6 ; Interleukin $17=I L-17$; Interleukin 17=IL-23, Sharp-van der Heijde score( modified total Sharp score, mTSS), joint erosions=JE, joint space narrowing=JSN,: Health Assessment Questionnaire=HAQ,short form 36 health questionnaire=SF-36,Disease Activity Score $=$ DAS, $15 \mathrm{~m}$ walking time $=15 \mathrm{mWT}$.

Data management: NoteExpree (V3.0) was used to perform data management. Two researchers independently screened titles and abstracted for potentially relevant studies after eliminating duplications. Full texts were downloaded and read for eligible included studies based on inclusion and exclusion criteria. Then a cross-check after completion was performed to avoid mis-entry. Any discrepancies were discussed by a third reviewer. Data extraction was independently completed by two researchers. Discrepancies were discussed with a third reviewer. A data 
extraction form was developed in advance. The main contents of data extraction were as follows: - General information (title, author, country, retrieved database, and language). - Study characteristics (numbers of included RCTs, quality assessment tool, interventions and comparisons, data analysis methods). • Outcomes. - Summary of conclusions.

Quality assessment / Risk of bias analysis: Assessment of Methodological Quality. AMSTAR 2 is a common instrument to evaluate the methodology of SRs. Two researchers independently assessed the methodology of SRs using AMSTAR2. AMSTAR2 has 16 items, including seven critical items (item 2/4/7/9/11/13/15) which can critically affect the validity of a SR and its conclusion. Each item was evaluated as "yes," "partial yes," and "no" according to the standard of AMSTAR 2 guideline.Discrepancies were solved by team discussion. Assessment of Risk of Bias. ROBIS is a tool designed specifically to assess the risk of bias in SRs, which is comprised of three phases formed by signaling questions. Two researchers independently assessed the risk of bias of each SR using ROBIS. Phase 1 assesses the assessing relevance, which is optional. Phase 2, which is comprised of four domains formed by 21 signaling questions, aims to identify concerns with the review process. Phase 3, with three signaling questions, concentrates to judge risk of bias of the SR. All signaling questions were answered as "yes", "probably yes", "probably no", "no", and "no information". If all of signaling questions of phase 3 were answered as "yes," SR was judged as "low risk". Any of signaling question of phase 3 was answered as "probably no" or "no", SR was assessed as "high risk". If the information provided was insufficient to judge, SR was rated as "unclear risk". Discrepancies were solved by team discussion.

Strategy of data synthesis: Our study aimed to conduct a comprehensive overview and to critically appraise the methodology, risk of bias, reporting quality, and quality of evidence of these SRs, by using the tools of $A$ Measurement Tool to Assess Systematic Reviews 2 (AMSTAR 2), Risk of bias in systematic reviews (ROBIS), Preferred Reporting Items for Systematic reviews and Meta-Analyses (PRISMA), and the grading of recommendations assessment, development, and evaluation (GRADE), respectively. Besides, the effectiveness and safety of TWHF for RA were narratively summarized.

Subgroup analysis: None.

Sensitivity analysis: None.

Language: Studies published in English and Chinese were included.

Country(ies) involved: All authors are from China.

Other relevant information: All authors have experience in participating in systematic reviews.

Keywords: Tripterygium wilfordii Hook.f preparations, Rheumatoid arthritis, AMSTAR 2, ROBIS, PRISMA, GRADE, overview of systematic reviews.

Dissemination plans: Published in the magazine and communicated with the conference.

Contributions of each author:

Author 1 - Li Huimin - The author drafted the manuscript.

Email: oklihuimin@163.com

Author 2 - Hu Ruixue - The authors Huimin $\mathrm{Li}$ and Ruexue $\mathrm{Hu}$ contributed equally to this work.

Author 3 - Dai Zeqi - The author contributed to the development of the selection criteria, and the risk of bias assessment strategy.

Email: daizq_1015@163.com

Author 4 - Xu Simin - The author read, provided feedback and approved the final manuscript.

Email: 1620344120@qq.com

Author 5 - Liao Xing -The author provided all the Articles.

Email: okfrom2008@hotmail.com 